\title{
Increased Expression of Tenascin-X in Thoracic and Abdominal Aortic Aneurysm Tissues
}

\author{
Kazumi Satoh, ${ }^{a}$ Marie Tsukamoto, ${ }^{a}$ Masanobu Shindoh,${ }^{b}$ Yasunori Totsuka, ${ }^{c}$ Teiji Oda, ${ }^{d}$ and \\ Ken-ichi Matsumoto*,a \\ ${ }^{a}$ Department of Biosignaling and Radioisotope Experiment, Center for Integrated Research in Science, Shimane \\ University; ${ }^{d}$ Division of Cardiovascular and Thoracic Surgery, Department of Surgery, Faculty of Medicine, Shimane \\ University; Enya-cho, Izumo 693-8501, Japan: ${ }^{b}$ Department of Oral Pathology and Biology, Graduate School of Dental \\ Medicine, Hokkaido University; and ${ }^{c}$ Department of Oral and Maxillofacial Surgery, Graduate School of Dental \\ Medicine, Hokkaido University; Sapporo 060-8586, Japan.
}

Received July 24, 2010; accepted August 24, 2010; published online August 26, 2010

Tenascin-X (TNX), which has a molecular mass of roughly $450 \mathrm{kDa}$, is the largest member of the tenascin family. Complete deficiency of TNX in humans leads to a recessive form of Ehlers-Danlos syndrome (EDS). TNX is expressed abundantly in a variety of tissues, especially in cardiac muscle and in perivascular stroma. Human TNX is also present in serum with an apparent molecular size of $140 \mathrm{kDa}$. In the present study, we investigated the expression levels of TNX protein in thoracic and abdominal aortic aneurysm tissues. The level of TNX was significantly increased in both aortic aneurysm tissues compared with that in adjacent normal tissues. Next, to compare TNX levels in serum from both patients with thoracic aortic aneurysm and patients with abdominal aortic aneurysm with levels in serum from healthy individuals, we developed a sandwich enzyme-linked immunosorbent assay (ELISA) using TNX-specific antibodies. Measurement of TNX serum concentrations in both aortic aneurysm patients and controls showed that the levels were almost the same. These results indicate that TNX expression is significantly elevated in both thoracic and abdominal aortic aneurysm tissues but that the increase in TNX levels in both tissues does not result in an increase in TNX serum concentration in patients with TAA or AAA.

Key words tenascin-X; thoracic aortic aneurysm; abdominal aortic aneurysm; sandwich enzyme-linked immunosorbent assay

Tenascins are large oligomeric glycoproteins of the extracellular matrix $(\mathrm{ECM})$. So far, four members of the tenascin family have been identified in vertebrates: tenascin-C (TNC), tenascin-R (TNR), tenascin-X (TNX) (known as tenascin- $\mathrm{Y}$ in birds), and tenascin-W (TNW). ${ }^{1)}$ Tenascin family members have the same types of structural domains, including a cysteine-rich segment at the amino terminus, epidermal growth factor (EGF)-like repeats, fibronectin type III (FNIII)-like repeats, and a fibrinogen (FG)-like domain at the carboxyl terminus.

TNX is the largest member of the tenascin family with about $450 \mathrm{kDa}$. Complete deficiency of TNX in humans leads to a rare recessive form of Ehlers-Danlos syndrome (EDS), and TNX haploinsufficiency is associated with hypermobility type of EDS. ${ }^{2)}$ The skin of TNX-deficient patients is markedly lax with poor recoil properties and shows easy bruising. TNX-deficient mice partly mimic this phenotype. ${ }^{3)}$ Several observations suggest that TNX is involved in collagen fibrillogenesis, ${ }^{4,5)}$ collagen deposition, ${ }^{6}$ ) modulation of collagen stiffness, ${ }^{7)}$ and development and maintenance of elastic fibers. ${ }^{8)}$ The serum form of TNX has also been identified in humans and mice.9,10)

There are several lines of evidence suggesting that an aneurysm results from a localized inflammatory process leading to complex remodeling that involves both the synthesis and degradation of extracellular matrix (ECM) components in the aortic wall. Infiltrating macrophages release matrix metalloproteinases (MMPs), particularly MMP-9 and MMP-2. MMPs are a family of proteolytic enzymes that cause degradation of various components of the ECM and participate in vascular remodeling. Structural integrity of the aortic wall depends to a large extent on ECM components in- cluding elastin and collagen. ${ }^{11)}$ The role of MMPs in the pathogenesis of degenerative abdominal aortic aneurysms (AAAs) and thoracic aortic aneurysms (TAAs) is well established. ${ }^{12)}$ The role of TNC in the development of abdominal $^{13,14)}$ and thoracic ${ }^{15,16)}$ aortic aneurysms has also been reported. Furthermore, an association of AAA with TNX expression has been shown. ${ }^{17)}$

It is known that an aortic aneurysm exhibits extensive increases of wall vascularization and that its pathogenesis is attributed to neovascularization of the aortic wall. Previously, we showed that TNX in combination with vascular endothelial growth factor B (VEGF-B) enhances endothelial cell proliferation. ${ }^{18)}$ Serum TNX also binds to VEGF-A and VEGF$\mathrm{B}$ and possesses weak activity for proliferation of endothelial cells. ${ }^{19)}$ TNC has been observed in the stroma around blood vessels that are undergoing angiogenesis in AAAs. ${ }^{14)}$ These findings suggest that the tenascin family plays important roles in the neovascularization of aortic wall in abdominal and thoracic aortic aneurysms.

In this study, we investigated the expression of TNX protein in TAA and AAA tissues. Furthermore, we measured serum TNX concentration using a sandwich enzyme-linked immunosorbent assay (ELISA) for TNX in patients with TAA or AAA. We found that TNX protein is highly expressed in both aortic aneurysm tissues compared to the expression level in adjacent normal tissues. However, serum TNX concentrations are almost the same in patients with aortic aneurysm and healthy individuals.

\section{MATERIALS AND METHODS}

Patients and Samples The participants gave informed 
consent, and the studies were performed with the approval of the Ethics Committee of Shimane University School of Medicine, Japan. Aortic aneurysm tissue was obtained at surgery from subjects with 11 TAAs and from subjects with 15 AAAs who underwent elective aneurysm repair. Reference samples were obtained from normal aorta tissues adjacent to TAA or AAA tissues.

For ELISA assays for determination of serum TNX concentration, blood samples were obtained from 8 TAA patients, 10 AAA patients and 12 adult healthy volunteers. Each tissue and serum sample was snap-frozen in liquid nitrogen and stored at $-80^{\circ} \mathrm{C}$ until protein extraction and use for ELISA assays, respectively.

Western Blot Analysis About $20 \mathrm{mg}$ of tissue samples was washed with cold phosphate-buffered saline (PBS) and homogenized in lysis buffer containing $50 \mathrm{~mm}$ Tris- $\mathrm{HCl}(\mathrm{pH}$ 7.5), $150 \mathrm{~mm} \mathrm{NaCl}, 10 \mathrm{~mm}$ ethylenediaminetetraacetic acid (EDTA), $20 \mathrm{~mm}$ urea, $1 \mathrm{~mm}$ phenylmethylsulfonyl fluoride (PMSF), and Complete ${ }^{\mathrm{TM}}$ protease inhibitor set (Roche Diagnostics, Indianapolis, IN, U.S.A.). The homogenate was centrifuged and the supernatant was collected and stored at $-80^{\circ} \mathrm{C}$. The protein concentration was determined by a bicinchoninic acid (BCA) assay (Pierce, Rockford, IL, U.S.A.). Equal amounts of sample proteins were mixed with Laemmli buffer, boiled for $5 \mathrm{~min}$, and subjected to $10 \%$ sodium dodecyl sulfate-polyacrylamide gel electrophoresis (SDS-PAGE) under reducing conditions for Western blot analysis. Western blot analysis was performed as previously described. ${ }^{20)}$ After electrophoretic transfer of proteins to enhanced chemiluminescence (ECL) nitrocellulose filters (GE Healthcare, Buckinghamshire, England), the filters were reacted with antibodies against affinity-purified rabbit antiTNX ( $\alpha$-hu30) polyclonal antibodies, with rabbit anti-VEGF polyclonal antibodies (Calbiochem, Cambridge, MA, U.S.A.), or with mouse anti-actin monoclonal antibody (Millipore, Temecula, CA, U.S.A.). The anti-VEGF antibodies detect a VEGF-A ${ }_{165}$ isoform as shown in the manufacturer's sheet. Then the filters were washed three times with TBST containing $20 \mathrm{~mm}$ Tris- $\mathrm{HCl}(\mathrm{pH} 7.5), 150 \mathrm{~mm} \mathrm{NaCl}$ and $0.1 \%$ Tween-20 and incubated with horseradish peroxidase (HRP)-labeled anti-rabbit or anti-mouse immunoglobulin G (IgG) (MBL, Nagoya, Japan) followed by visualization using the ECL Plus Western Blotting Detection System (GE Healthcare, Hino, Japan). Quatification of the intensity of each band was performed using free and open source ImageJ software. In the case of immunostaining with anti-TNX antibodies, several immunoreactive bands that represent proteolytic products of $\mathrm{TNX}^{10)}$ appeared as shown in Fig. 1A. Therefore, total of intensity of each band was calculated and used for quantification of TNX level.

ELISA Assay for Measurement of Serum TNX Concentration For the preparation of human TNX-specific antibodies, $\alpha$-hu30 and $\alpha$-hu31, for antigen capture and antigen detection, respectively, recombinant hu30 and hu31 proteins (antigens) encoding the 30th and the 31 st FNIII repeats of human TNX were purified, as described previously. ${ }^{10)}$ For an ELISA standard curve, a recombinant hu30-31 protein encoding from the 30th FNIII repeat to the 31st FNIII repeat of human TNX was purified. For a negative standard, a recombinant hW4 protein that encodes the 3rd FNIII repeat of human TNW was purified. In brief, for the hu30 protein, forward fhu30 primer 5'-GGGGATCCGGGCTGGCTCCTGCT-3' and reverse rhu30 primer 5'-GGGAATTCTGGGCTGAGGGT-3' as a primer set and Image clone IMAGp998A0513619Q3 as a DNA template were used for polymerase chain reaction (PCR). Similarly, for the hu31 protein, forward fhu31 primer 5'-GGGGATCCGTTCTGGAGAGCCCC-3' and reverse rhu31 primer 5'-GGGAATTCCGTGGTGAGGAAGCC-3' as a primer set and Image clone IMAGp998A0513619Q3 as a DNA template were used for PCR, and for the hu30-31 protein, forward fhu30 primer and reverse rhu31 primer as a primer set and Image clone IMAGp998A0513619Q3 as a DNA template were used for PCR. For the hW4 protein, forward fhTNW4 primer 5'-GGGGATCCCTCCTGAATGGCAGG-3' and reverse rhTNW4 primer 5'-GGGAATTCCCCCTGGTTGCCCCT-3' as a primer set and NBRC clone AK127044 (National Institute of Technology and Evaluation, Tokyo, Japan) as a DNA template were used for PCR. The PCR products were cloned into pGEX-6p-3 vector (GE Healthcare), and the recombinant plasmids were transformed into Escherichia coli BL21 (DE3) and the bacteria were induced to express the recombinant protein in the presence of isopropyl- $\beta$-D-thiogalactopyranoside (IPTG) for $4 \mathrm{~h}$ at $30^{\circ} \mathrm{C}$. After lysis of the bacteria, glutathione-S-transferase (GST)-fusion proteins were extracted and subsequently purified using GlutathioneSepharose 4B beads (GE Healthcare). For GST-free proteins, the GST tag was cleaved using PreScission Protease (GE Healthcare) as shown previously. ${ }^{10)}$ Subsequently, female Japanese White rabbits were immunized 6 times with hu30 and hu31 proteins. Finally, human TNX-specific rabbit polyclonal antibodies, $\alpha$-hu30 and $\alpha$-hu31 were affinity-purified from the antiserum according to our previously described method. ${ }^{10)}$ The specificity of the antibody labeling was confirmed by Western blot analysis.

For TNX detection in the ELISA experiment, $\alpha$-hu31 antibodies were labeled with horseradish peroxidase kits according to the instructions of the manufacturer (Dojindo Molecular Technologies, Kumamoto, Japan). Microtiter plates (Thermo Scientific, Yokohama, Japan) were coated with rabbit $\alpha$-hu30 antibodies $\left(1 \mu \mathrm{g} / \mathrm{ml}\right.$ in PBS) overnight at $4{ }^{\circ} \mathrm{C}$. After washing with PBS containing $0.05 \%$ Tween 20 (PBST), PBST with $2.5 \%$ milk was added to each well to block non-specific binding sites. After 1-h incubation at room temperature (around $24^{\circ} \mathrm{C}$ ), the wells were washed six times with PBST and then incubation was performed for a further $1.5 \mathrm{~h}$ at room temperature after addition of 5-fold di-

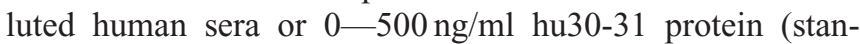
dard). Then the wells were washed nine times with PBST, incubated with horseradish peroxidase-conjugated $\alpha$-hu31 antibodies $(0.3 \mu \mathrm{g} / \mathrm{ml})$ for $1.5 \mathrm{~h}$ at room temperature, washed nine times, and incubated with a chromogenic substrate solution (DAKO TMB Blue) (DAKO, Carpinteria, CA, U.S.A.) for $3 \mathrm{~min}$. The reaction was stopped by addition of $1 \mathrm{M}$ $\mathrm{H}_{2} \mathrm{SO}_{4}$. Then absorbance was measured at $450 \mathrm{~nm}$ by EMax Precision Microplate Reader (Molecular Devices, Sunnyvale, CA, U.S.A.). The standard curve was plotted on the semi-log axes with the log-logit fit in SoftMax Pro Software (Molecular Devices).

Statistical Analysis Results are expressed as means S.E. Differences were compared using the unpaired $t$-test or Mann-Whitney $U$-test after analysis of variance. 

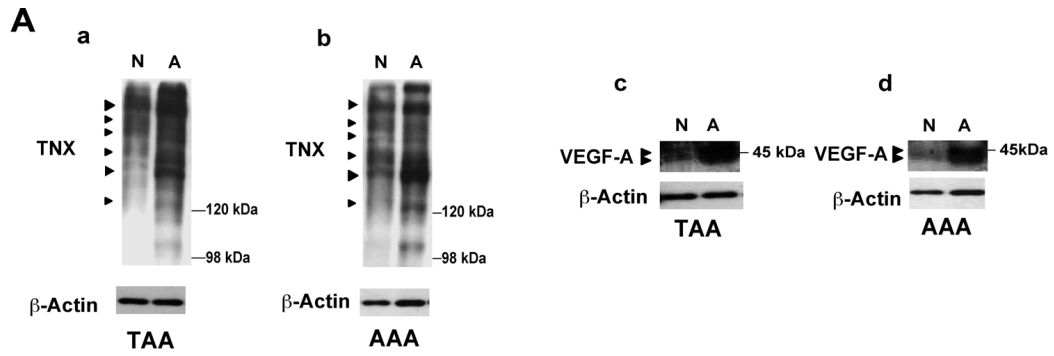

B
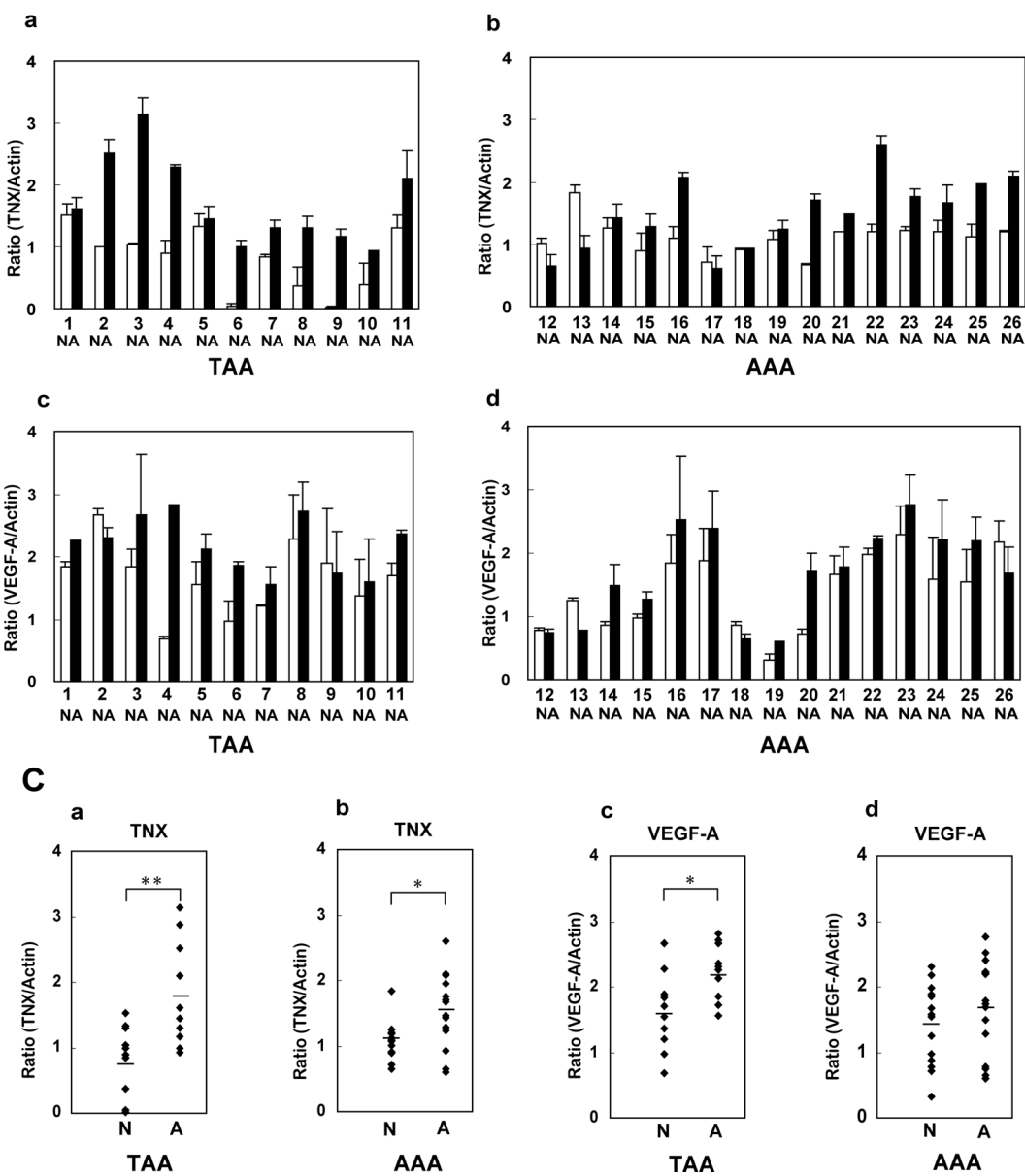

Fig. 1. Expression Profiles of TNX and VEGF-A in 11 AAA and 15 TAA Tissues

(A) Immunoblot analyses of TAA tissues and AAA tissues from patients. Lysates from TAA tissues (patients \#7) were immunoblotted with anti-TNX antibodies ( $\alpha$-hu30 antibodies) (a) and anti-VEGF-A antibodies (c). Similarly, lysates from AAA tissues (patients \#14) were immunoblotted with anti-TNX antibodies (b) and anti-VEGF-A antibodies (d). Lane "A" indicates each sample derived from aortic aneurysm tissues, whereas lane " $N$ " indicates that from adjacent normal aorta tissues. Immunostaining of the human samples with anti-TNX antibodies showed several immunoreactive bands (arrowheads) that represent proteolytic products of TNX as found previously in mouse tissues. ${ }^{10)}$ (B) Statistical analyses of TNX and VEGF-A in 11 AAA and 15 TAA tissues. Lysates from each of the TAA tissues (\#1-\#11) $(n=11)$ were immunoblotted with anti-TNX antibodies and antiVEGF-A antibodies. Similarly, lysates from each of the AAA tissues $(\# 12-\# 26)(n=15)$ were immunoblotted with anti-TNX antibodies and anti-VEGF-A antibodies. Statistical analysis was performed for ratio of TNX level to $\beta$-actin level in each TAA (a) and each AAA (b) tissues (lane A) and for ratio of VEGF-A level to its $\beta$-actin level for each TAA tissue (c) and each AAA tissue (d) (lane A) compared with those in adjacent normal aorta tissues (lane N). Note that very low level of TNX expression is observed in the normal tissues (N) of patients \#6 and \#9 (Fig. 1Ba). This might be due to the distinctive feature of these normal tissues. The Western blot experiment was repeated $2-3$ times with one homogenate from each of TAA, AAA and adjacent normal aorta tissues, and means \pm S.E. of experiments were calculated and shown. (C) Distribution of TNX and VEGF-A levels in 11 AAA and 15 TAA tissues. TNX/ $\beta$-actin levels in each of the TAA (a) and AAA (b) tissues (lane A) as shown in Figs. 1Ba and 1Bb and VEGF-A/ $\beta$-actin levels in each of the TAA (c) and AAA (d) tissues (lane A) as shown in Figs. 1Bc and 1Bd compared with those in adjacent normal aorta tissues (lane N) are presented. Data represent the mean \pm S.E. $* * p<0.01, * p<0.05$ by unpaired $t$-test; significantly different from the adjacent normal aorta tissues.

\section{RESULTS}

Increased Expression of TNX in TAA and AAA Tissues To investigate whether the expression of TNX protein is altered in TAA (patient \#7) and AAA (patient \#14) tissues compared with that in adjacent normal aorta tissues, Western blot analysis was performed using the TNX-specific antibody $\alpha$-hu30. As shown in Figs. 1Aa and 1Ab, TNX is up-regu- lated in TAA and AAA tissues (lane A) compared with that in adjacent normal aorta tissues (lane $\mathrm{N}$ ). To confirm this result statistically, 11 TAA and 15 AAA tissues were examined. As shown in Figs. $1 \mathrm{Ba}, 1 \mathrm{Bb}, 1 \mathrm{Ca}$, and $1 \mathrm{Cb}$, statistical analysis by using ImageJ software was performed on densitometry readings of the ratio of TNX level to $\beta$-actin level for each TAA tissue (Figs. $1 \mathrm{Ba}, 1 \mathrm{Ca}$ ) and each AAA tissue (Figs. 1Bb, 1Cb) (lane A) compared with that in adjacent 
normal aorta tissues (lane N). TAA tissues $(n=11)$ had a ratio of TNX level to $\beta$-actin level (1.71) greater than that in adjacent normal aorta tissues $(0.79)(p=0.0029)$, and AAA tissues $(n=15)$ had a ratio of TNX level to $\beta$-actin level (1.49) slightly larger than that in adjacent normal aorta tissues $(1.10)(p=0.027)$. These statistical analyses indicated that TNX level is significantly increased in both TAA and AAA tissues compared with that in adjacent normal aorta tissues.

It is known that active angiogenesis persists in aortic aneurysms. ${ }^{14)}$ Furthermore, TNX binds to VEGF family members, leading to an enhancement of endothelial cell proliferation. ${ }^{18,19)}$ Therefore, we investigated the expression level of VEGF-A in each TAA tissue and each AAA tissue (lane A) compared with that in adjacent normal aorta tissues (lane $\mathrm{N}$ ), as shown in Figs. 1Ac (patient \#7), 1Ad (patient \#14), $1 \mathrm{Bc}, 1 \mathrm{Bd}, 1 \mathrm{Cc}$, and $1 \mathrm{Cd}$. Statistical analysis showed that TAA tissues $(n=11)$ had a ratio of VEGF-A level to $\beta$-actin level (2.18) greater than that in adjacent normal aorta tissues (1.64) $(p=0.022)$. However, the same analysis showed no distinct differences in VEGF-A level relative to $\beta$-actin level between 15 AAA tissues (1.67) and adjacent normal aorta tissues (1.39) $(p=0.25)$. These results indicated that VEGFA level is significantly increased in TAA tissues but not in AAA tissues.

Serum TNX Concentrations in TAA and AAA Patients Next, to investigate whether the increased levels of TNX in TAA and AAA tissues have an influence on serum concentration of TNX in patients with TAA or AAA, we developed a sandwich ELISA method to measure serum level of TNX in the patients. As shown in Fig. 2A, a standard curve for a recombinant human TNX protein, hu30-31, in this ELISA was obtained in a dose-dependent manner. On the other hand, GST protein or a recombinant human TNW protein, hW4 (negative control protein for ELISA experiments), was not detected in the ELISA system regardless of the concentrations of recombinant proteins. Thus, we concluded that this ELISA system is useful for the measurement of serum level of TNX in humans.

The serum levels of TNX in 12 control healthy volunteers, 8 TAA patients, and 10 AAA patients are shown in Fig. 2B. The mean TNX concentrations in the TAA and AAA patient

A

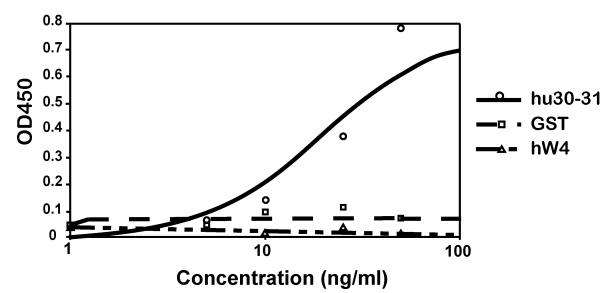

\section{B}

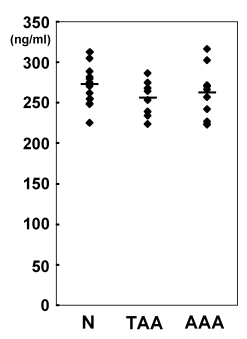

Fig. 2. Measurement of Serum TNX Concentrations in TAA and AAA Patients

(A) Standard curve of sandwich ELISA using a recombinant human TNX protein, hu30 - 31, was obtained by a sandwich ELISA using purified anti-TNX antibodies $(\alpha-$ hu30 antibodies for antigen capture, HRP-labeled $\alpha$-hu31 antibodies for antigen detection). As negative controls, GST protein and a recombinant human TNW protein, hW4, were used for this ELISA system. The standard curve was plotted on the semi-log axes with the log-logit fit in SoftMax Pro Software. (B) Distribution of serum TNX levels in the TAA and AAA groups of patients compared with that in the normal control group (N). The experiment was repeated 6 times. Data represent the mean. groups were $255.6 \pm 7.7 \mathrm{ng} / \mathrm{ml}$ (mean \pm S.E., $n=8$ ) and $260.1 \pm 10.2 \mathrm{ng} / \mathrm{ml}(n=10)$, respectively, whereas the mean concentration in the control group was $271.2 \pm 7.2 \mathrm{ng} / \mathrm{ml}$ $(n=12)$. No statistical difference was found between TNX levels in the control group and the TAA and AAA patient groups by Mann-Whitney $U$-test analysis. These results indicated that the increase in TNX levels in TAA and AAA tissues does not lead to an increase in serum TNX concentration in these patients.

\section{DISCUSSION}

In initial works for identification of possible circulating ECM biomarkers, it was shown that the aminoterminal propeptide of type III procollagen (PIIINP) ${ }^{21,22)}$ is associated with AAA by measuring their plasma concentrations. However, recent studies have shown that there is no association of plasma PIIINP level with AAA. ${ }^{23)}$ On the other hand, as another possible circulating ECM biomarker, Zweers and colleagues ${ }^{17)}$ reported higher serum concentrations of TNX in 87 patients with AAA compared with those in 86 control samples. They showed that TNX serum concentration in the highest quartile was associated with a 5-fold increased risk of AAA (odds ratio, 5.3; 95\% confidence interval, 2.0 to 13.8).

In this study we found that TNX levels in TAA and AAA tissues were increased by 2.2 fold $(p=0.0029)$ and 1.4 fold $(p=0.027)$, respectively, compared with those in adjacent normal tissues. However, no significant difference was found between serum TNX concentrations in the control group and TAA or AAA patient group. Thus, the increase in TNX level in TAA and AAA tissues did not lead to an increase in serum TNX concentration in these patients. It seems that increase in TNX in the aneurysmal wall does not give rise to accelerated turnover and/or leakage of TNX from the wall. Zweers and colleagues ${ }^{17)}$ investigated TNX expression in 5 AAA tissues and 5 normal aorta samples by an immunostaining method. In contrast to our results, they reported that TNX expression is markedly decreased in AAA tissues. A possible explanation for this discrepancy is the technical difference for detection of TNX protein, namely, by immunostaining technique in Zweers' study, and by Western blot analysis followed by statistical quantification in our study. The Western blot method used in this study is more sensitive for quantification of protein level than the immunostaining techniques used in previous studies. Furthermore, Zweers and colleagues also found a slight difference between serum TNX concentrations in the control group and AAA patient group (control group: median, $378 \mathrm{ng} / \mathrm{ml}$; range, $269-657 \mathrm{ng} / \mathrm{ml}$; AAA patient group: median, $422 \mathrm{ng} / \mathrm{ml}$; range, $251-602 \mathrm{ng} / \mathrm{ml} ; p<0.02$ ). A possible explanation for the disparity between their findings and our results is that the control serum samples in Zweers' study were from patients with diabetes, heart, vascular disease and/or pulmonary disease. Our control samples for measurement of serum TNX concentration were obtained from 12 healthy volunteers without any disease. Furthermore, the discrepancy in ELISA data between Zweers' study and our result might be due to the difference of TNX antibodies used in the experiments. They used rabbit polyclonal antibodies for antigen capture and guinea pig antibodies for antigen detection, both raised against a $100-\mathrm{kDa}$ C-terminal 
fragment of human TNX expressed in E. coli. ${ }^{9,17)}$ On the other hand, we used human TNX-specific antibodies, $\alpha$-hu30 for antigen capture and $\alpha$-hu31 for antigen detection, raised against the 30 th and the 31 st FNIII repeats of human TNX, respectively.

Since we previously demonstrated that TNX in combination with VEGF family members enhances endothelial cell proliferation, ${ }^{18,19)}$ we thought it would be interesting to know whether TNX expression is associated with expression of VEGF-A in TAA and AAA tissues, in which the level of TNX is increased. As expected, VEGF-A level was significantly increased in TAA tissues $(p=0.022)$, in coincidence with the increased expression of TNX, but not in AAA tissues. This result suggests that TNX is involved in angiogenesis in combination with VEGF family members in TAA tissues.

In conclusion, serum levels of TNX do not correlate with their tissue levels in TAA and AAA. Thus, they cannot be used as a serum marker of the TNX degradation process in the aneurysmal wall. However, there are also limitations of this study that must be acknowledged. The sample size in this study is relatively small. Furthermore, we should take into account confounding by unknown factors to influence our results. Further detailed studies are still necessary to prove the value of TNX as a circulating extracellular matrix marker for TAA and/or AAA.

Acknowledgments We thank Tomoko Toga for her technical assistance and Hitomi Arauchi for her contribution to preparing some figures. This work was supported in part by Grants-in-Aid for Scientific Research from the Ministry of Education, Culture, Sports, Science and Technology of Japan and by Grants of Research for Promoting Technological Seeds from Japan Science and Technology Agency.

\section{REFERENCES}

1) Tucker R. P., Drabikowski K., Hess J. F., Ferralli J., Chiquet-Ehrismann R., Adams J. C., BMC Evol. Biol., 6, 1-17 (2006).

2) Zweers M. C., Hakim A. J., Grahame R., Schalkwijk J., Arthritis
Rheum., 50, 2742-2749 (2004).

3) Mao J. R., Taylor G., Dean W. B., Wagner D. R., Afzal V., Lotz J. C., Rubin E. M., Bristow J., Nat. Genet., 30, 421-425 (2002).

4) Minamitani T., Ikuta T., Saito Y., Takebe G., Sato M., Sawa H., Nishimura T., Nakamura F., Takahashi K., Ariga H., Matsumoto K., Exp. Cell Res., 298, 305-315 (2004).

5) Egging D., van den Berkmortel F., Taylor G., Bristow J., Schalkwijk J., Arch. Dermatol. Res., 298, 389-396 (2007).

6) Veit G., Hansen U., Keene D. R., Bruckner P., Chiquet-Ehrismann R., Chiquet M., Koch M., J. Biol. Chem., 281, 27461-27470 (2006).

7) Margaron Y., Bostan L., Exposito J. Y., Malbouyres M., TrunfioSfarghiu A. M., Berthier Y., Lethias C., Biophys. Chem., 14, 87—91 (2010).

8) Zweers M. C., van Vlijmen-Willems I. M., van Kuppevelt T. H., Mecham R. P., Steijlen P. M., Bristow J., Schalkwijk J., J. Invest. Dermatol., 122, 885-891 (2004).

9) Schalkwijk J., Zweers M. C., Steijlen P. M., Dean W. B., Taylor G., van Vlijmen I. M., van Haren B., Miller W. L., Bristow J., N. Engl. J. Med., 345, 1167-1175 (2001).

10) Matsumoto K., Kinoshita T., Hirose T., Ariga H., DNA Cell Biol., 25, $448-456$ (2006).

11) MacSweeney S. T., Powell J. T., Greenhalgh R. M., Br. J. Surg., 81, 935-941 (1994).

12) Hobeika M. J., Thompson R. W., Muhs B. E., Brooks P. C., Gagne P. J., J. Vasc. Surg., 45, 849-857 (2007).

13) Satta J., Soini, Y., Pöllänen R., Pääkkö P., Juvonen T., J. Vasc. Surg., 26, 670-675 (1997).

14) Paik D. C., Fu C., Bhattacharya J., Tilson M. D., Exp. Mol. Med., 36, 524-533 (2004).

15) Nataatmadja M., West M., West J., Summers K., Walker P., Nagata M., Watanabe T., Circulation, 108 (Suppl. II), II329-II334 (2003).

16) Majumdar R., Miller D. V., Ballman K. V., Unnikrishnan G., McKellar S. H., Sarkar G., Sreekumar R., Bolander M. E., Sundt T. M. 3rd., Cardiovasc. Pathol., 16, 144-150 (2007).

17) Zweers M. C., Peeters A. C., Graafsma S., Kranendonk S., van der Vliet J. A., den Heijer M., Schalkwijk J., Circulation, 113, 17021707 (2006).

18) Ikuta T., Ariga H., Matsumoto K., Genes Cells, 5, 913-927 (2000).

19) Ishitsuka T., Ikuta T., Ariga H., Matsumoto K., Biol. Pharm. Bull., 32, 1004-1011 (2009).

20) Nakamura Y., Takayama N., Minamitani T., Ikuta T., Ariga H., Matsumoto K., Gene, 251, 55-62 (2000).

21) Satta J., Juvonen T., Haukipuro K., Juvonen M., Kairaluoma M. I., $J$ Vasc. Surg., 22, 155-160 (1995).

22) Treska V., Topolcan O., Int. Angiol., 19, $64-68$ (2000).

23) Eugster T., Huber A., Obeid T., Schwegler I., Gürke L., Stierli P., Eur. J. Vasc. Endovasc. Surg., 29, 378-382 (2005). 